\title{
Effect of resveratrol on non-alcoholic fatty liver disease
}

\author{
MARIOS THEODOTOU ${ }^{1,2}$, KONSTANTINOS FOKIANOS $^{3}$, DEMETRIS MONIATIS ${ }^{2}$, \\ RUDOLF KADLENIC ${ }^{2}$, ASIMINA CHRYSIKOU ${ }^{2}$, ANDREA ARISTOTELOUS ${ }^{2}$, \\ ALEXIA MOUZOURIDOU $^{4}$, JOHN DIAKIDES $^{2}$ and ELIZA STAVROU ${ }^{2}$
}

\author{
${ }^{1}$ Private Practice; ${ }^{2}$ Riegler Ltd., Institute of Medical Clinical Trials, Limassol 3020; ${ }^{3}$ Department of Mathematics and \\ Statistics, University of Cyprus, Nicosia 20537; ${ }^{4}$ Agios Efrem, Advanced Medical Diagnostic Center, Limassol 3020, Cyprus
}

Received September 14, 2018; Accepted October 26, 2018

DOI: $10.3892 /$ etm.2019.7607

\begin{abstract}
The aim of the present study was to investigate the effect of a micronized formulation of trans-resveratrol in humans with non-alcoholic fatty liver disease (NAFLD). Trans-Resveratrol has been used in the form of micronized formulation, which is better absorbed, has strong antioxidants effects, is more effective than plain resveratrol formulations and is circulated on the market as a food supplement. Resveratrol (3,5,4'-trihydroxy-trans-stilbene) is a stilbenoid and a phytoalexin produced by several plants. NAFLD is an increasing clinical problem involving the liver for which effective treatments are required. The present study was based on two patient groups. The study, which commenced on April 2013 and finished on April 2015, included 44 patients, aged 29-70 years, with an average weight of $84.6 \mathrm{~kg}(\mathrm{n}=22$ per group; 28 men and 16 women) who were randomly assigned to groups and given $50 \mathrm{mg}$ Evelor capsule $(\mathrm{n}=22)$ and $200 \mathrm{mg}$ Evelor $\mathrm{H}$ tablet $(\mathrm{n}=22)$ correspondingly on a daily basis. The patients were followed up for 6 months. Quantity fat measurements, with ultrasound on the liver and kidney, were carried out. There was an initial measurement (time 1) and one after six months (time 2). The study results showed the effects of Trans-resveratrol micronized formulation in reducing the liver fat, as well as decreasing hepatic enzymes, serum glutamate pyruvic transaminase (SGPT) and gamma-glutamyl transpeptidase (g-GT) and insulin resistance. At the end of the study, the statistical analysis showed a statistically significant reduction on the liver fat. These data demonstrate that use of Trans-resveratrol micronized formulation improves features of NAFLD, and prevents liver damage. Thus, Trans-resveratrol micronized formulation can be a new treatment method for NAFLD.
\end{abstract}

Correspondence to: Dr Marios Theodotou, Riegler Ltd., Institute of Medical Clinical Trials, Leontiou A 181, Limassol 3020, Cyprus E-mail:info@mariostheodotou.com

Key words: liver, resveratrol, non-alcoholic fatty liver disease, hepatic enzymes, liver value

\section{Introduction}

Non-alcoholic fatty liver disease (NAFLD) is the most common liver disease worldwide. It affects up to $30 \%$ of adults in Western countries and $15 \%$ in Asian countries and also an increasing number of children (1). NAFLD is a clinical syndrome characterized by the accumulation of excess fat in the liver. It spans a spectrum of disease from pathological accumulation of triglyceride (TG), steatosis, to an inflammatory response, non-alcoholic steatohepatitis (NASH) (2). NASH may progress to cirrhosis, cirrhosis complications, liver failure and an increased risk of liver cancer (3). NAFLD is the third cause of liver transplantation in the United States (4).

NAFLD is becoming a major health issue worldwide, not only for its prevalence, but also for its metabolic complications. The underlying insulin resistance is associated with hypertension, hyperlipidemia, cardiovascular disease, type 2 diabetes mellitus (T2DM), chronic kidney disease and recently with carotid atherosclerosis (5-7). Therapeutic options are limited, there is no pharmacological therapy and managing NAFLD focuses on the treatment of risk factors.

The polyphenol resveratrol (RSV) is a potential therapeutic candidate. RSV is a stilbenoid and a phytoalexin produced by several plants in response to injury or when the plant is under attack by pathogens such as bacteria or fungi (8). It is found mainly in Japanese knotweed, red grapes and in other plants, in low concentrations (8). During the last decades, the potential of RSV has been explored. It has pleiotropic effects in various tissues. RSV is an activator of adenosine monophosphate-activated kinase (AMPK) and silent information regulation 2 homolog 1 (SIRT1). The two proteins have a critical role in aiding fat breakdown and removal from the liver, associated with liver diseases such as fibrosis and cirrhosis (9). Through the activation of AMPK and SIRT1 in hepatic cells and anti-oxidant and anti-inflammatory actions, RSV may prevent liver damage and may inhibit the progression of NAFLD $(10,11)$.

Through clinical practice, it has been found that, in patients who suffer from arterial hypertension and elevated hepatic enzymes, treated with anti-hypertensive drugs and a strong antioxidant, such as micronized trans-resveratrol, hepatic enzymes were significantly improved. Thus, it was decided to start a clinical trial regarding the effect of micronized trans-resveratrol in patients with Non-alcoholic fatty liver disease (NAFLD). 
In the present study, 50 or $200 \mathrm{mg}$ RSV (Evelor and Evelor H, a food supplement manufactured by Agetis Supplements Ltd., Cyprus) was added to the standard treatment of patients with NAFLD daily for a period of 6 months and the therapeutic efficacy of RSV was investigated.

\section{Materials and methods}

Patient characteristics. The study was approved by the Ethics Committee of Cyprus (file no. ЕЕВK/ЕП/2010/12, date 06/06/2013). All the patients who participated were volunteers and they were asked to fill out a written consent form.

The patients were selected after they had initially been diagnosed with non-alcoholic fatty liver disease. The primary inclusion criterion was evidence of fatty liver on ultrasonography (US), which is the most commonly used imaging technique with remarkable sensitivity.

The US findings in non-alcoholic fatty liver disease are: i) Diffuse enhancement of near field echo in the hepatic region (stronger than in the kidney and in the spleen region) and gradual attenuation of the far field echo, ii) unclear display of intra-hepatic lacuna structure, iii) mild to moderate hepatomegaly with a round and blunt border, and iv) unclear display of right liver lobe and diaphragm (12).

The study excluded patients with one or more of the following features: i) Any known causes of steatosis, ii) cirrhosis, ii) malignant tumor or any other diseases which significantly decrease the patient's lifespan, iii) symptoms of heart failure or acute coronary syndrome, iv) chronic kidney disease, v) psychiatric disorders, vi) participating in other similar studies or participating in other studies that were completed in the last 6 months, and vii) using food supplements, they should stop the supplement and wait for two weeks before participating in the study.

Equipment used. A GE LogIQ5 expert Ultrasound Machine (General Electric, CA, USA), equipped with Ultrasound Transducer Probe (GE 3.5C model 2050357) was used. The equipment can measure the Echo Level (EL) at specific areas and depths. EL measures the mean intensity of pixels within a user-defined area (region of interest). Raw data provide the average sum (intensity per pixel)/pixels. The ultrasound depicts on screen the area (in $\mathrm{cm}^{2}$ ), mean (intensity $\mathrm{dB}$ ) and standard deviation (dispersion). Phantom Model 040GSE (CIRS-Multi Purpose, Multi Tissue u/s Phantom) was used to calibrate the LogIQ5 machine. The specific phantom simulates the human liver and kidney organs and serves for calibration.

Regarding the parameters 'liver/kidney value' and 'liver/kidney depth', we used the same methodology used by Xia et al (13). The procedure was as follows: An experienced radiologist, who was unaware of the patient's clinical details and laboratory findings, performed ultrasound studies. All the instrument settings, including gain and depth were fixed for each measurement. For assessment of the ultrasound Hepatic/Renal echo value, ultrasound images with both liver and right kidney clearly visualized were obtained in the sagittal liver/right kidney view in the lateral position. A region of interest (ROI) was carefully selected excluding blood vessels, bile ducts and other focal hypoechoic or hyperechoic regions. Another ROI was identified in the right renal cortex with no large vessels, renal sinus or medulla. To avoid the interference of depth-dependent echo-intensity attenuation and the borderline echo distorting effects, the boundary between liver and right kidney area was placed near the center of the image, and the liver and right kidney ROIs were selected at the same depth of the ultrasound images. The gray scale mean value of the pixels within the two ROIs was used as measurement of echo intensity, followed by subtraction of the average hepatic gray scale by the average renal cortex gray scale to calculate the US hepatic/renal value.

Standardization of ultrasound quantitative parameters was performed using an abdominal phantom.

Method of calculation. Attenuation measurements were taken at 2 depths, at the ROI xxcm (liver) and yycm (kidney). Attenuation was calculated by subtracting the EL (liver)-EL (kidney)=Hepato-Renal Index Difference. EL was measured in $\mathrm{dB}$ and was linear to the intensity; thus, linear regression was employed to compute normalized values (14).

US hepatic/renal echo value. In sagittal liver/right kidney view, an ROI of $1.5 \times 1.5 \mathrm{~cm}(1,296$ pixels $)$ in the liver parenchyma was selected. The ROI had to be as uniform as possible, excluding blood vessels, bile ducts, and other focal hypo/hyper echogenicity. Another ROI of $0.5 \times 0.5 \mathrm{~cm}$ (144 pixels) was identified in the right renal cortex with no large vessels, renal sinus or medulla. To avoid the interference of depth-dependent echo-intensity attenuation and the borderline echo distorting effects, the boundary between liver and right kidney area was placed near the center of the image, and the liver and right kidney ROIs were selected at the same depth of the ultrasound images. The gray scale mean value of the pixels within the two ROIs was used as measurement of echo intensity. Then we subtracted the average hepatic gray scale from the average renal cortex gray scale to calculate the US hepatic/renal value.

US hepatic echo-intensity attenuation rate. In the right intercostal view at the anterior axilla line, a tangent line of the sector ultrasound image was drawn and the ultrasound wave transmission line was determined, starting from the point of tangency and perpendicular to the tangent line. Two ROIs of $1.5 \times 1.5 \mathrm{~cm}(1,296$ pixels $)$ were selected in liver homogeneous regions along the ultrasound transmission line near the liver anterior margin (depth, 4-6 cm) and the liver posterior margin, respectively. The linear distance between the two ROIs was also measured. The echo intensity of the ultrasound wave was attenuated exponentially, as shown in the equation:

$$
A_{\mathrm{d}}=A_{0} \times \mathrm{e}^{-a \cdot f \cdot d}
$$

where $A_{0}$ and $A_{\mathrm{d}}$ are the ultrasound echo intensity at the sound source and the liver parenchyma at a specific depth, respectively; $a$ is the attenuation coefficient of the liver parenchyma; $f$ is the frequency of the ultrasound detector; $d$ is the depth of ROI. 
The ratio of the average echo intensity in the liver near-field ROI to liver far-field ROI was then calculated based on the equation 1:

$$
A_{\mathrm{n}} / A_{\mathrm{f}}=\mathrm{e}^{a \cdot f \cdot(\mathrm{d} f-\mathrm{d} n)}
$$

where $A_{\mathrm{n}}$ and $A_{\mathrm{f}}$ are average ultrasound echo intensity in the near-field ROI and the far-field ROI, respectively; $a$ and $f$ have been defined in equation $1 ; \mathrm{d} n$ and $\mathrm{d} f$ are the depth of liver near-field and far-field ROIs.

Then the formula for ultrasound hepatic echo-intensity attenuation rate was deduced from the equation 2 :

$$
a=\left(\ln A_{\mathrm{n}}-\ln A_{\mathrm{f}}\right) /(\Delta d \cdot f)
$$

where $\Delta d$ is the distance between the near-field and far-field ROIs, and other parameters are defined in equation 2.

Standardization of ultrasound quantitative parameters. To standardize the measured values of US H/R value and hepatic echo-intensity attenuation rate among different ultrasound machines, a 3D abdominal phantom, containing mimic abdominal organs, was used for standardization in this research.

Treatment method. Participants underwent clinical examination, electrocardiogram and abdominal ultrasound. Blood tests were carried out and the following parameters were measured: Serum glutamic pyruvic transaminase (SGPT), serum glutamic oxaloacetic transaminase (SGOT), alkaline phosphatase (ALP), gamma-glutamyl transpeptidase (g-GT), glucose, total cholesterol levels, high-density lipoprotein (HDL), low-density lipoprotein (LDL), triglycerides (TG), insulin, insulin resistance, liver value, liver depth, kidney value, kidney depth and difference liver-kidney values.

Then, the patients were divided into two groups (randomly assigned) according to the treatment administered: 22 patients were given treatment A (Evelor, $50 \mathrm{mg}$ RSV) and 22 patients were given treatment B (Evelor H, $200 \mathrm{mg}$ RSV). The observation period lasted for 6 months. There was a clinical examination, blood tests and an abdominal ultrasound at the beginning of the study (time 1) and in 6 months (time 2). All the patients were on low fat diet and were followed up by a nutritionist.

The results obtained by the study were used to examine the following parameters: i) The count of hepatic enzymes, ii) insulin resistance, and iii) liver fat.

Statistical analysis. Two-way ANOVA and Bonferroni post-hoc test, with interaction (time and group) was used to determine whether there were differences among dose levels, time levels and a possible interaction among them. However, we did not discover any significant interactions and we used standard two-way ANOVA without interactions. Moreover, an independent t-test was conducted to examine whether there were any differences between the values of physiological parameters at the beginning and the end of the study. $\mathrm{P}<0.001$ was considered to indicate a strongly statistically significant difference. The statistical program used for statistical analysis was R3.2.1.

\section{Results}

Subjects. A total of 44 patients participated in the study; 28 men and 16 women from the ages of 29 to 70 with a mean weight of $84.6 \mathrm{~kg}$. The patients were divided into two groups of 22 individuals each, according to the treatment administered: Group A was treated with micronized trans-Resveratrol $50 \mathrm{mg}$ (Evelor), and Group B was treated with micronized trans-Resveratrol $200 \mathrm{mg}$ (Evelor $\mathrm{H}$ ). Measurements were made at the beginning (time 1) and after 6 months (time 2) to compare results between the two different dose groups as well as within the group itself. Tables I and II contain summary statistics for all the participants in the study with a focus on the main patient demographics including age, weight and height.

Table III shows the mean values of all the variables in the beginning (time 1) and after 6 months (time 2) of the study and the results of comparisons among different time-points for those participants in the $50 \mathrm{mg}$ group. We obtained the following conclusions: i) There were strongly significant differences among liver values that were initially at 55.20. In fact, the liver values decreased (time 2 ) resulting in a P-value of $<0.001$. ii) There were strongly significant differences among kidney values that were initially at 32.49. In fact, the kidney values decreased (time 2) resulting in a P-value of $<0.001$. iii) There were significant differences among insulin resistance that were initially at 1405.81 . In fact the insulin resistance values decreased (time 2) resulting in a P-value of 0.135 .

Table IV contains the mean values of all the variables in the beginning (time 1), and after six months (time 2) of the study, the results of comparisons among different time-points for those participants in the $200 \mathrm{mg}$ group. We obtained the following conclusions: i) There were strongly significant differences among liver values that were initially at 58.77. In fact, the liver values decreased (time 2) resulting in a P-value of $<0.001$. ii) There were strongly significant differences among kidney values that were initially at 32.30 . In fact, the kidney values decrease (time 2 ) resulting in a P-value of $<0.001$. iii) There were significant differences among insulin resistance that were initially at 1541.04 . In fact the insulin resistance values decrease (time 2) resulting in a P-value of 0.151 .

Table $\mathrm{V}$ contains the comparisons among groups and different time points for all participants in the study. We used a two-way ANOVA model with interaction to test whether there are differences among dose levels, time levels and possible interaction among them. We obtained the following conclusions: i) There are strongly significant differences between liver values and kidney values across the time, interaction between time and dose (p-value) 0.383 for liver value and 0.778 for kidney value. Both of these measurements decreased (time 2). ii) The difference between liver and kidney values decreased as time progressed. iii) There were statistically significant differences between the two dose levels for ALP and TG $(\mathrm{P}<0.05)$. iv) There were significant differences between the dose levels for SGOT, glucose and HDL.

Based on the fact that there was no interaction between dose and time for all variable considered, we also implemented a two-way ANOVA model but without interaction. Table VI contains the comparisons among groups and different time points for all the participants in the study. 
Table I. Main demographic variables.

\begin{tabular}{|c|c|c|c|c|c|}
\hline Variables & Mean & SD & Median & Minimum & Maximum \\
\hline Age (years) & 54.16 & 9.92 & 55 & 29 & 70 \\
\hline Weight $(\mathrm{kg})$ at time 1 & 84.55 & 11.42 & 83.30 & 58 & 105 \\
\hline Weight $(\mathrm{kg})$ at time 2 & 82.33 & 11.73 & 80 & 57 & 103 \\
\hline Height $(\mathrm{cm})$ & 170 & 7 & 170 & 155 & 183 \\
\hline BMI $\left(\mathrm{kg} / \mathrm{cm}^{2}\right)$ at time 1 & 27.01 & 3.02 & 28 & 20 & 31 \\
\hline BMI $\left(\mathrm{kg} / \mathrm{cm}^{2}\right)$ at time 2 & 27.93 & 3.35 & 28.50 & 21 & 35 \\
\hline
\end{tabular}

BMI, body mass index.

Table II. Classification of the participants by sex and treatment.

\begin{tabular}{lcc}
\hline Sex/Dose & $50 \mathrm{mg}$ RSV & $200 \mathrm{mg}$ RSV \\
\hline Male & 12 & 16 \\
Female & 10 & 6 \\
\hline
\end{tabular}

RSV, resveratrol.

Table III. Statistical comparisons based only on $50 \mathrm{mg}$ (Evelor) dose across time.

\begin{tabular}{lrrr}
\hline Variables & $\begin{array}{c}\text { Mean at } \\
\text { Time 1 }\end{array}$ & $\begin{array}{c}\text { Mean at } \\
\text { Time 2 }\end{array}$ & $\begin{array}{c}\text { t-test } \\
\text { (P-value) }\end{array}$ \\
\hline SGPT & 36.09 & 37.24 & 0.871 \\
SGOT & 24.95 & 26.33 & 0.603 \\
ALP & 63.63 & 59.23 & 0.593 \\
g-GT & 30.95 & 27.00 & 0.770 \\
GLU & 105.95 & 96.57 & 0.122 \\
CH & 194.50 & 185.67 & 0.656 \\
HDL & 48.60 & 47.67 & 0.905 \\
LDL & 119.45 & 116.19 & 0.932 \\
TG & 132.50 & 109.76 & 0.301 \\
Insulin & 13.58 & 12.69 & 0.206 \\
Insulin resistance & 1405.81 & 1226.04 & 0.135 \\
Liver value & 55.20 & 45.42 & $<0.001$ \\
Liver depth & 5.60 & 5.79 & 0.034 \\
Kidney value & 32.49 & 28.42 & $<0.001$ \\
Kidney depth & 7.06 & 7.30 & 0.008 \\
Difference L-K value & 22.71 & 17.00 & 0.170 \\
\hline
\end{tabular}

SGPT, serum glutamate pyruvic transaminase; SGOT, serum glutamic oxaloacetic transaminase; ALP, alkaline phosphatase; g-GT, gamma-glutamyl transpeptidase; GLU, glucose; HDL, high-density lipoprotein; LDL, low-density lipoprotein; TG, triglycerides.

The results show the same conclusions as in the case of a model that includes interactions: i) There were strongly statistically significant differences between Liver value and Kidney value across time. Both of these measurements, liver
Table IV. Statistical comparisons based only on $200 \mathrm{mg}$ (Evelor $\mathrm{H}$ ) dose across time.

\begin{tabular}{lrrr}
\hline Variables & $\begin{array}{c}\text { Mean at } \\
\text { Time 1 }\end{array}$ & $\begin{array}{c}\text { Mean at } \\
\text { Time 2 }\end{array}$ & $\begin{array}{c}\text { t-test } \\
\text { (P-value) }\end{array}$ \\
\hline SGPT & 47.05 & 41.70 & 0.631 \\
SGOT & 32.81 & 30.90 & 0.887 \\
ALP & 74.50 & 71.20 & 0.804 \\
g-GT & 29.20 & 25.35 & 0.434 \\
GLU & 121.18 & 112.50 & 0.786 \\
CH & 194.27 & 181.15 & 0.258 \\
HDL & 41.14 & 41.70 & 0.832 \\
LDL & 118.75 & 105.95 & 0.408 \\
TG & 205.13 & 167.80 & 0.465 \\
Insulin & 12.62 & 13.01 & 0.091 \\
Insulin resistance & 1541.04 & 1489.52 & 0.151 \\
Liver value & 58.77 & 43.33 & $<0.001$ \\
Liver depth & 5.20 & 5.63 & 0.792 \\
Kidney value & 32.30 & 25.21 & $<0.001$ \\
Kidney depth & 7.04 & 7.26 & 0.795 \\
Difference L-K value & 26.46 & 18.115 & 0.795 \\
\hline
\end{tabular}

SGPT, serum glutamate pyruvic transaminase; SGOT, serum glutamic oxaloacetic transaminase; ALP, alkaline phosphatase; g-GT, gamma-glutamyl transpeptidase; GLU, glucose; HDL, high-density lipoprotein; LDL, low-density lipoprotein; TG, triglycerides.

value and kidney value decreased (time 2). ii) The difference liver-kidney decreased as time progressed. iii) There were statistically significant differences between the two dose levels for ALP and TG. iv) There are significant differences between the dose levels for SGOT, glucose and HDL.

\section{Discussion}

Liver is an important organ because of its unique metabolism. Its main function is to take up nutrients, to store and/or provide them to the other organs. At the same time, it is a clearance and recycling organ. That means it is also a frequent target for a number of toxicants. The resulting hepatic injury is characterized by leakage of cellular enzymes into the blood stream. 
Table V. Statistical comparisons based on different doses and across time.

\begin{tabular}{lccc}
\hline Variables & $\begin{array}{c}\text { Time effect } \\
\text { (P-value) }\end{array}$ & $\begin{array}{c}\text { Dose effect } \\
\text { (P-value) }\end{array}$ & $\begin{array}{c}\text { Interaction between } \\
\text { time and dose (P-value) }\end{array}$ \\
\hline SGPT & 0.842 & 0.052 & 0.576 \\
SGOT & 0.991 & 0.022 & 0.686 \\
ALP & 0.563 & $<0.001$ & 0.883 \\
g-GT & 0.567 & 0.377 & 0.798 \\
GLU & 0.336 & 0.005 & 0.963 \\
CH & 0.215 & 0.563 & 0.863 \\
HDL & 0.983 & 0.004 & 0.764 \\
LDL & 0.479 & 0.190 & 0.697 \\
TG & 0.183 & $<0.001$ & 0.898 \\
Insulin & 0.027 & 0.782 & 0.887 \\
Insulin resistance & 0.021 & 0.179 & 0.914 \\
Liver value & $<0.001$ & 0.621 & 0.383 \\
Liver depth & 0.136 & 0.821 & 0.376 \\
Kidney value & $<0.001$ & 0.287 & 0.778 \\
Kidney depth & 0.051 & 0.330 & 0.350 \\
Difference L-K value & 0.001 & 0.681 & 0.424 \\
\end{tabular}

SGPT, serum glutamate pyruvic transaminase; SGOT, serum glutamic oxaloacetic transaminase; ALP, alkaline phosphatase; g-GT, gamma-glutamyl transpeptidase; GLU, glucose; HDL, high-density lipoprotein; LDL, low-density lipoprotein; TG, triglycerides.

Table VI. Statistical comparisons based on different doses and across time.

\begin{tabular}{lrr}
\hline Variables & $\begin{array}{r}\text { Time effect } \\
\text { (P-value) }\end{array}$ & $\begin{array}{r}\text { Dose effect } \\
\text { (P-value) }\end{array}$ \\
\hline SGPT & 0.841 & 0.051 \\
SGOT & 0.991 & 0.022 \\
ALP & 0.556 & $<0.001$ \\
g-GT & 0.563 & 0.374 \\
GLU & 0.330 & 0.004 \\
CH & 0.211 & 0.460 \\
HDL & 0.983 & 0.004 \\
LDL & 0.475 & 0.187 \\
TG & 0.179 & $<0.001$ \\
Insulin & 0.025 & 0.781 \\
Insulin resistance & 0.020 & 0.176 \\
Liver value & $<0.001$ & 0.621 \\
Liver depth & 0.136 & 0.821 \\
Kidney value & $<0.001$ & 0.284 \\
Kidney depth & 0.051 & 0.331 \\
Difference L-K value & 0.001 & 0.681 \\
\hline
\end{tabular}

SGPT, serum glutamate pyruvic transaminase; SGOT, serum glutamic oxaloacetic transaminase; ALP, alkaline phosphatase; g-GT, gamma-glutamyl transpeptidase; GLU, glucose; HDL, high-density lipoprotein; LDL, low-density lipoprotein; TG, triglycerides.

Elevation of SGPT, SGOT, g-GT in the blood often reflects hepatocellular damage $(15,16)$.
Normal ranges of SGOT/SGPT in blood are 5-40 or 7-56 U/1 and these are sensitive indicators of liver damage from different types of diseases. SGPT is primarily located in the liver and represents more specifically an injury to the organ as compared to SGOT, which is found in decreasing order of concentration in liver, cardiac muscle, skeletal muscle, kidneys, lungs, and brain.

Gamma-GT normal range in blood is 5-55 U/1. It is primarily present in kidney, liver, and pancreatic cells. Small amounts are present in other tissues. Even though renal tissue has the highest level of g-GT, the enzyme present in the serum appears to originate primarily from the hepatobiliary system, and g-GT activity is elevated in any and all forms of liver disease. It is currently the most sensitive enzymatic indicator of liver disease (17).

The common causes of elevated SGPT, SGOT, g-GT are alcohol abuse, drugs, chronic hepatitis $\mathrm{B}$ and $\mathrm{C}$, autoimmune hepatitis, congenital metabolic disorders and fatty liver disease. In the United States, the majority of unexplained cases of elevated transaminases are strongly associated with non-alcoholic fatty liver disease (NAFLD) (18).

NAFLD is the most common form of chronic liver disease and a major health burden in developed countries with a prevalence of up to $30 \%$ (19). Two thirds of the patients are asymptomatic. It is characterized by the accumulation of triglycerides in the liver and spans a histological spectrum of liver disease, ranging from simple steatosis to steatohepatitis, fibrosis and rarely to cirrhosis or even hepatocellular carcinoma (20). It was considered to be a benign condition, but is now recognized as an important cause of liver-related morbidity and mortality (20). 
Recent studies have shown an association between NAFLD and metabolic syndrome, as it seems to have a common pathogenic factor, insulin resistance (IR) (21). Major expressions of NAFLD are diabetes mellitus type II and obesity. NAFLD has also been associated with acute starvation, total parenteral nutrition, abdominal surgery, use of several drugs and chemicals, and rare metabolic disorders (19). Depending on the pathogenesis, NAFLD is classified as, primary NAFLD associated with insulin resistance and secondary NAFLD associated with other conditions (22).

Although clinical studies have tried several pharmacological treatments, there is currently no satisfactory therapy for NAFLD. Therefore, investigators focus on the management of metabolic syndrome (22). Previous findings demonstrated that, weight loss by calorie restriction (CR), improves insulin resistance and fatty acid metabolism $(23,24)$ and it is the only effective treatment for NAFLD. However, the long-term adherence to lifestyle modifications is hard. Alternative treatments are therefore required.

Natural polyphenols are a potential therapeutic option for NAFLD. They have been proposed for the treatment of different metabolic disorders, because of their anti-inflammatory and anti-oxidative properties. Additionally, they have an effect on glucose and lipid metabolism (24).

The polyphenolresveratrol(RSV3,5,4'-trihydroxystilbene) is a stilbenoid produced by several plants in response to injury or when the plant is under attack by pathogens. RSV has multiple biochemical and physiological actions. One of the most important is that RSV mimics a condition of caloric restriction (CR) (25) and this could be beneficial for the treatment of NAFLD. Previous findings have indicated that RSV improves insulin sensitivity, reduces insulin-like growth factor 1 (IGF-I) levels and activates key regulators of metabolism, such as adenosine monophosphate-activated kinase (AMPK) and silent information regulator 1 (SIRT1) (26).

AMPK is a protein consisting of three subunits, one catalytic and two non-catalytic. AMPK is activated, by phosphorylation, as a response to changes in the cellular AMP/ATP ratio (27). Findings have shown that RSV promoted the phosphorylation of AMPK. Once activated, AMPK regulates the lipid and glucose metabolism. AMPK suppresses anabolic processes and promotes catabolic processes. It reduces the activities of lipogenesis-associated genes, such as sterol regulatory element-binding protein-1c $(S R E B P-1 c)$ and fatty acid synthase $(F A S)$, leading to reduced lipogenesis and lipid accumulation (28). It also inactivates acetyl-CoA carboxylase (ACC) and promotes the activity of carnitine palmitoyltransferase-1 (CPT-1) and this leads to a decrease of liver fat accumulation (29). AMPK is a key molecule in the pathogenesis of NAFLD. Its other metabolic effect is that it promotes glucose metabolism, as it inhibits gluconeogenesis and enhances glucose uptake in the skeletal muscle.

RSV is also an activator of SIRT1, which is a NAD+ (oxidized nicotinamide adenine dinucleotide)-dependent protein deacetylase. SIRT1 plays a key role in lipid and glucose homeostasis and in insulin secretion sensitivity via CR. Furthermore, SIRT1 is an inhibitor of inflammation, reduces oxidative stress and improves endothelial function (30). In the liver, deacetylates activate certain proteins resulting in increased fatty acid $\beta$-oxidation (31). A number of studies have confirmed that activation of SIRT1 affects the pathogenetic molecular cascade of NAFLD $(30,32,33)$.

In this study, the results indicated that trans-resveratrol in micronized formulation supplementation prevents and improves liver damage. The mechanism that RSV mimics CR is not fully understood yet, but the activation of AMPK and SIRT1 has a key role. The reduction of TG accumulation and the improvement of IR serve to protect the liver from NAFLD. Therefore, our data suggest that trans-resveratrol in micronized formulation is a hepatoprotective agent in humans, and a new therapeutic option for NAFLD.

\section{Acknowledgements}

Not applicable

\section{Funding}

This study was financially supported by the 'Program Sustainable Development and Competitiveness'.

\section{Availability of data and materials}

The datasets used and/or analyzed during the current study are available from the corresponding author on reasonable request.

\section{Authors' contributions}

MT designed and performed the study. AC, AA and AM were responsible for the collection and analysis of the data. KF, DM and RK performed data analysis and interpretation. JD and ES participated in analysing the data and revised the manuscript. All authors read and approved the final manuscript.

\section{Ethics approval and consent to participate}

All patients provided written informed consent for the use of their data. The study protocol was approved by the Cyprus National Bioethics Committee.

\section{Patient consent for publication}

Not applicable.

\section{Competing interests}

The authors declare that they have no competing interests.

\section{References}

1. VernonG,BaranovaAandYounossiZM:Systematicreview:Theepidemiology and natural history of non-alcoholic fatty liver disease and non-alcoholic steatohepatitis in adults. Aliment Pharmacol Ther 34: 274-285, 2011.

2. Chalasani N, Younossi Z, Lavine JE, Diehl AM, Brunt EM, Cusi K, Charlton M and Sanyal AJ: The diagnosis and management of nonalcoholic fatty liver disease: Practice guideline by the American Association for the Study of Liver Diseases, American College of Gastroenterology, and the American Gastroenterological Association. Hepatology 55: 2005-2023, 2012. 
3. Chachay VS, Macdonald GA, Martin JH, Whitehead JP, O'Moore-Sullivan TM, Lee P, Franklin M, Klein K, Taylor PJ, Ferguson M, et al: Resveratrol does not benefit patients with nonalcoholic fatty liver disease. Clin Gastroenterol Hepatol 12: 2092-2103.e1-e6, 2014.

4. Charlton MR, Burns JM, Pedersen RA, Watt KD, Heimbach JK and Dierkhising RA: Frequency and outcomes of liver transplantation for nonalcoholic steatohepatitis in the United States. Gastroenterology 141: 1249-1253, 2011.

5. Bonora E and Targher G: Increased risk of cardiovascular disease and chronic kidney disease in NAFLD. Nat Rev Gastroenterol Hepatol 9: 372-381, 2012.

6. Angulo P: Nonalcoholic fatty liver disease. N Engl J Med 346: $1221-1231,2002$

7. Fracanzani AL, Burdick L, Raselli S, Pedotti P, Grigore L, Santorelli G, Valenti L, Maraschi A, Catapano A and Fargion S: Carotid artery intima-media thickness in nonalcoholic fatty liver disease. Am J Med 121: 72-78, 2008.

8. Frémont L: Biological effects of resveratrol. Life Sci 66: 663-673, 2000.

9. Kopec KL and Burns D: Nonalcoholic fatty liver disease: A review of the spectrum of disease, diagnosis, and therapy. Nutr Clin Pract 26: 565-576, 2011.

10. Lagouge M, Argmann C, Gerhart-Hines Z, Meziane H, Lerin C, Daussin F, Messadeq N, Milne J, Lambert P, Elliott P, et al: Resveratrol improves mitochondrial function and protects against metabolic disease by activating SIRT1 and PGC-1alpha Cell 127: 1109-1122, 2006.

11. Gómez-Zorita S, Fernández-Quintela A, Macarulla M Aguirre L, Hijona E, Bujanda L, Milagro F, Martínez JA and Portillo MP: Resveratrol attenuates steatosis in obese Zucker rats by decreasing fatty acid availability and reducing oxidative stress. Br J Nutr 107: 202-210, 2012

12. Zeng MD, Fan JG, Lu LG, Li YM, Chen CW, Wang BY and Mao YM; Chinese National Consensus Workshop on Nonalcoholic Fatty Liver Disease: Guidelines for the diagnosis and treatment of nonalcoholic fatty liver diseases. J Dig Dis 9: 108-112, 2008

13. Xia MF, Yan HM, He WY, Li XM, Li CL, Yao XZ, Li RK, Zeng MS and Gao X: Standaridized ultrasound hepatic/renal ratio and hepatic attenuation rate to quantify liver fat content: An improvement method. Obesity (Silver Spring) 20: 444-452, 2012

14. von Volkmann HL, Havre RF, Løberg EM, Haaland T, Immervoll $\mathrm{H}$, Haukeland JW, Hausken T and Gilja $\mathrm{OH}$ : Quantitative measurement of ultrasound attenuation and hepato-renal index in non-alcoholic fatty liver disease. Med Ultrason 15: 16-22, 2013.

15. Ramadori G, Moriconi F, Malik I and Dudas J: Physiology and pathophysiology of liver inflammation damage and repair. J Physiol Pharmacol 59 (Suppl 1): S107-S117, 2008.

16. Jaeschke H, Gores GJ, Cederbaum AI, Hinson JA, Pessayre D and Lemasters JJ: Mechanisms of hepatotoxicity. Toxicol Sci 65 $166-176,2002$

17. Tietz Textbook of clinical chemistry. Edited by Burtis CA Ashwood ER and Saunders WB Company, Philadelphia, 1994.

18. Clark JM, Brancati FL and Diehl AM: The prevalence and etiology of elevated aminotransferase levels in the United States. Am J Gastroenterol 98: 960-967, 2003.

19. Bedogni G, Miglioli L, Masutti F, Tiribelli C, Marchesini G and Bellentani S: Prevalence of and risk factors for nonalcoholic fatty liver disease: The Dionysos nutrition and liver study. Hepatology 42: 44-52, 2005.
20. Adams LA, Lymp JF, St Sauver J, Sanderson SO, Lindor KD, Feldstein A and Angulo P: The natural history of nonalcoholic fatty liver disease: A population-based cohort study. Gastroenterology 129: 113-121,2005.

21. Paschos P and Paletas K: Nonalcoholic fatty liver disease and metabolic syndrome. Hippokratia 13: 9-19, 2009.

22. Duvnjak M, Lerotić I, Barsić N, Tomasić V, Virović Jukić L and Velagić V: Pathogenesis and management issues for non-alcoholic fatty liver disease. World J Gastroenterol 13: 4539-4550, 2007.

23. Alberti KG, Zimmet $P$ and Shaw J; IDF Epidemiology Task Force Consensus Group: The metabolic syndrome-a new worldwide definition. Lancet 366: 1059-1062, 2005.

24. Ahmed MH and Byrne CD: Modulation of sterol regulatory element binding proteins (SREBPs) as potential treatments for non-alcoholic fatty liver disease (NAFLD). Drug Discov Today 12: 740-747, 2007.

25. Baur JA, Pearson KJ, Price NL, Jamieson HA, Lerin C, Kalra A, Prabhu VV, Allard JS, Lopez-Lluch G, Lewis K, et al: Resveratrol improves health and survival of mice on a high-calorie diet. Nature 444: 337-342, 2006

26. Price NL, Gomes AP, Ling AJ, Duarte FV, Martin-Montalvo A, North BJ, Agarwal B, Ye L, Ramadori G, Teodoro JS, et al: SIRT1 is required for AMPK activation and the beneficial effects of resveratrol on mitochondrial function. Cell Metab 15: 675-690, 2012

27. Yang YM, Han CY, Kim YJ and Kim SG: AMPK-associated signaling to bridge the gap between fuel metabolism and hepatocyte viability. World J Gastroenterol 16: 3731-3742, 2010.

28. Li Y, Xu S, Mihaylova MM, Zheng B, Hou X, Jiang B, Park O, Luo Z, Lefai E, Shyy JY, et al: AMPK phosphorylates and inhibits SREBP activity to attenuate hepatic steatosis and atherosclerosis in diet-induced insulin-resistant mice. Cell Metab 13: 376-388, 2011.

29. Alberdi G, Rodríguez VM, Macarulla MT, Miranda J, Churruca I and Portillo MP: Hepatic lipid metabolic pathways modified by resveratrol in rats fed an obesogenic diet. Nutrition 29: 562-567, 2013.

30. Colak Y, Ozturk O, Senates E, Tuncer I, Yorulmaz E, Adali G, Doganay L and Enc FY: SIRT1 as a potential therapeutic target for treatment of nonalcoholic fatty liver disease. Med Sci Monit 17: HY5-HY9, 2011.

31. Purushotham A, Schug TT, Xu Q, Surapureddi S, Guo X and Li X: Hepatocyte-specific deletion of SIRT1 alters fatty acid metabolism and results in hepatic steatosis and inflammation. Cell Metab 9: 327-338, 2009.

32. Li L, Hai J, Li Z, Zhang Y, Peng H, Li K and Weng X: Resveratrol modulates autophagy and $\mathrm{NF}-\kappa \mathrm{B}$ activity in a murine model for treating non-alcoholic fatty liver disease. Food Chem Toxicol 263: 166-173, 2014

33. Castro RE,Ferreira DM, Afonso MB, Borralho PM, Machado MV Cortez-Pinto $\mathrm{H}$ and Rodrigues CM: miR-34a/SIRT1/p53 is suppressed by ursodeoxycholic acid in the rat liver and activated by disease severity in human non-alcoholic fatty liver disease. J Hepatol 58: 119-125, 2013.

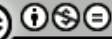

This work is licensed under a Creative Commons Attribution-NonCommercial-NoDerivatives 4.0 International (CC BY-NC-ND 4.0) License. 